\title{
El uso de la televisión en el hogar y en el aula de Educación Primaria
}

\section{The use of television at home and at the Primary School}

\author{
LOIDA M. LÓPEZ-MONDÉJAR ${ }^{1}$ \\ Ilopez@ucam.edu \\ Universidad Católica de Murcia, España
}

\section{Resumen:}

El presente artículo expone los resultados obtenidos en una investigación llevada a cabo en el alumnado de Educación Primaria sobre el consumo que se realiza del medio televisivo en el hogar, así como sobre el uso que se hace del mismo en el aula escolar. El estudio ha sido desarrollado en diferentes centros educativos de la Región de Murcia con alumnado ( $\mathrm{N}=320)$ de entre 9 y 10 años de edad y está enmarcado dentro de una investigación de carácter descriptivo donde se ha utilizado la técnica de encuesta. Los resultados muestran que la televisión sigue siendo uno de los medios más utilizados por el alumnado de Educación Primaria en el hogar, señalada como segunda actividad más deseada después de 'jugar con los amigos'. También sacan a la luz la tendencia generalizada, por parte del profesorado, de escaso uso de la televisión en el aula para el desarrollo de contenidos curriculares, así como la falta de trabajo con los alumnos sobre los contenidos visualizados. Siendo la televisión uno de los medios de comunicación más utilizados por los niños, este escaso uso que se realiza de la misma desde el aula escolar evidencia la gran separación entre escuela y sociedad y, por tanto, el absurdo

\begin{abstract}
:
This article presents the results obtained in a research carried out with pupils of Primary School regarding their television consumption both at home and at school. The study was developed in different schools of the Region of Murcia (in Spain) with pupils $(N=320)$ between 9 and 10 years of age and it is framed within a descriptive research where a survey method was used. The results show that television continues to be one of the most used media by pupils of Primary School at home. In fact, it is highlighted as the second most desired activity after 'playing with friends'. The data also revealed a generalized tendency: the scarce use of television in the classroom by the teachers for the development of the curricular contents, as well as the lack of analysis of the watched materials together with the students. Since television is one of the most used media by children, its limited use in the classroom evidences the great separation existing between school and society and, therefore, the absurdity that children live in relation to both their activities outside the classroom and the educational models and teaching-learning processes developed at school.
\end{abstract}

1 Dirección para correspondencia (correspondence address):

Loida López-Mondéjar. Facultad de Ciencias Sociales y de la Comunicación. Departamento de Educación. Universidad Católica de Murcia (UCAM). Campus de los Jerónimos, № 135. 30107 Guadalupe, Murcia (España). 
que los niños viven en relación a los modelos educativos y procesos de enseñanzaaprendizaje desarrollados en la escuela y sus actividades fuera de la misma.

\section{Palabras clave:}

Medios de comunicación de masas; televisión; educación en medios; Educación Primaria.

\section{Keywords:}

Mass media; television; media education; primary school.

\section{Résumé:}

Cet article présente les résultats obtenus grâce à une recherche menée auprès d'élèves de primaire, sur leur consommation de télévision à la fois à la maison et dans la salle de classe. L'étude a été mise au point dans différentes écoles de la région de Murcie (Espagne) avec des élèves $(N=320)$ âgés de 9 et 10 ans. Cette étude est encadrée dans une recherche descriptive où une méthode d'enquête a été utilisée. Les résultats montrent que la télévision est encore l'un des médias les plus utilisés par les élèves à la maison. D'ailleurs, il convient de noter qu'il s'agit de la deuxième activité la plus appréciée après "jouer avec des amis". Les données ont également révélé une tendance généralisée: la faible utilisation de la télévision en classe par les enseignants dans le but de développer les contenus des programmes, et l'absence d'une analyse des matériaux, ont été remarquées par les élèves. Puisque la télévision est l'un des médias les plus utilisés par les enfants, sa faible utilisation en classe met en évidence la grande séparation existant entre l'école et la société et, par conséquent, l'absurdité dans laquelle les enfants vivent. Il s'agit de mettre en relation leurs activités en dehors de l'école et les processus d'enseignementapprentissage mis au point dans les salles de classe.

\section{Mots clés:}

Médias de masse; télévision; éducation aux médias; école primaire.

Fecha de recepción: 30-5-2017

Fecha de aceptación: 20-6-2017

\section{Introducción y estado de la cuestión}

Los medios de comunicación son definidos por Middaugh y Kahne (2013) como un conjunto de instrumentos y contextos que permiten y cada vez adquieren más importancia para la participación de los ciudadanos. Además de ser herramientas que nos instruyen, también nos entretienen, abriéndonos puertas a lugares de conocimiento antes inimaginables (Núñez, 2009). Ballesta (2009) destaca de los medios su participación e influencia en todos los ámbitos de la población, ya sea en aspectos de su vida personal, en su tiempo de ocio o en el ámbito profesional. Subraya su carácter como transmisores de ideologías, valores, normas de comportamiento social y modelos culturales, así como señala su capacidad para homogeneizar hábitos y pensamientos. 
Una de las principales características que los define es la de ser uno de los más importantes emisores de contenidos e información de la sociedad, siendo su principal función la de acortar distancias y facilitar, a través de diferentes canales y vías, la comunicación e información entre los ciudadanos. Masterman (1993) los definía como 'sistemas simbólicos (o de signos) que necesitan ser leídos de manera activa y que no son reflejo incuestionable de la realidad externa ni se explican por sí mismos' (p. 36). Expresaba que, los medios como la televisión, la radio, los periódicos o el cine han sido producidos, esto es 'son agentes activos de los procesos de construcción o representación de la realidad' (p. 36), que no se limitan simplemente a transmitirla o reflejarla.

En este sentido conviene recordar la existencia de una concentración mediática en nuestra sociedad donde información y comunicación se combinan a través de las tecnologías, adquiriendo ambas cada vez un formato más interactivo, más atractivo y más cercano al usuario. De este modo representan, a la vez que les es otorgado, un papel de gran importancia en la vida de los ciudadanos, y ya no únicamente por su potencialidad como instrumentos o por los aspectos señalados previamente, sino por sus efectos y su gran influencia en relación a cómo desarrollamos una nueva interacción comunicativa individual y grupal a través de los mismos (Ballesta, Lozano y Cerezo, 2014). Por ello, los medios de comunicación se han convertido, según Sevillano (2012), en instituciones básicas dentro de nuestra sociedad, encargados de transmitir información, homogeneizar comportamientos, servir de sistemas para el equilibrio social, así como de ser el escenario de muchos de los problemas que presenta la sociedad.

Las tecnologías y medios de comunicación han modificado profundamente nuestra realidad social y cultural. Las nuevas formas de acceso a la información, con sus lenguajes propios, están desarrollando también nuevas formas de pensamiento en los ciudadanos de hoy, lo que lleva consigo nuevas formas de aprender, de acceso al conocimiento y de utilización de la información, llegando a cuestionarse el concepto de lo qué es y debe ser la institución escolar y los procesos de enseñanzaaprendizaje hoy día, aspectos que Ilevan consigo la necesidad de un cambio en los métodos utilizados en el proceso de enseñanza (Aguaded, 2010; Sevillano, 2012).

Los nuevos medios de comunicación están favoreciendo que los ciudadanos ya no accedan a un tipo de información lineal, sino a hiperme- 
dias, donde texto, imágenes, gráficos y vídeos se entrecruzan, y donde la interactividad, la inmediatez, la velocidad y el formato toman parte importante de la emisión y valoración del contenido informativo (Ballesta, 2010). Es por ello que resaltamos la idea de Burbules (2007), quien afirma que los cambios más importantes causados por las tecnologías y los medios de comunicación en nuestra sociedad y cultura no son producidos por las tecnologías y los medios en sí mismos, sino por un cambio en las ideas y en las prácticas sociales y culturales que los acompañan.

El concepto de 'alfabetización' en nuestra sociedad se ha ido modificando, ampliando y adaptando a los nuevos códigos, nuevos modos y nuevos medios de comunicación que han ido surgiendo a lo largo de la historia (Koltay, 2011). Así, mientras que en un principio el término tradicional de 'alfabetizado' era referido simplemente a aquella persona que sabía leer y escribir correctamente, en la actualidad, sin embargo, este significado ha sufrido una trasformación, abarcando el concepto un sentido más amplio.

De igual manera, al referirnos al término 'educación' en general, debemos tener presente que describe a aquella actividad o proceso inherente al ser humano que forma, dirige y desarrolla la vida de cada individuo con la finalidad de integrarlo en la sociedad en la que vive, convirtiéndolo en un ciudadano crítico, activo, democrático y participativo. Así, si actualmente nuestra sociedad se encuentra rodeada de multitud de mensajes provenientes de los distintos medios de comunicación, deberemos preparar a nuestros alumnos, con las capacidades, habilidades y actitudes que les permitan decodificar estos mensajes adecuadamente.

Cada vez más estudios demuestran que el consumo masivo de medios de comunicación que nuestros niños y jóvenes realizan en su día a día no lleva parejo un conocimiento de los códigos del lenguaje audiovisual adecuado, sino más bien al contrario, simplemente son consumidores de los mismos, lo que los sitúa en un escenario de total desamparo ante los continuos mensajes que de ellos van recibiendo. En este sentido, Álvarez (2012) advierte que aquellos a quienes denominamos nativos digitales, por el contrario, presentan demasiadas carencias o, como el autor expone, lo ignoran todo sobre aquello relacionado con ordenadores, redes y tecnología. Y es que los menores no disponen de una destreza natural para moverse en los nuevos entornos tecnológicos, sino que son sus necesidades comunicativas las que promocionan y propician 
ese uso, advierte De la Torre (2009). De ahí que podamos denominar a esta nueva generación de niños y jóvenes que han nacido y vivido entre las tecnologías de la información y los medios de comunicación como 'analfabetos audiovisuales' (Gómez y Aguaded, 2011) pues, aun haciendo un gran uso cotidiano de los mismos, no son conscientes de las repercusiones que su mala utilización puede tener en sus vidas y de cómo éstos pueden influenciarlos.

En este sentido, y conscientes del gran impacto que las tecnologías y los medios de comunicación tienen en nuestra sociedad, donde la pérdida de influencia cultural e ideológica de la institución educativa sobre la infancia y juventud es cada vez más destacada, siendo ésta inclinada a favor de los medios de comunicación, creemos necesario un cambio en los modelos educativos que tradicionalmente se vienen desarrollando en nuestras escuelas. Tal y como Aguaded y Guerra (2012) nos indican, el modelo de educación tradicional que se viene dando en los centros se encuentra cada vez más alejado de las nuevas exigencias que las nuevas generaciones presentan, siendo así necesario una adaptación al entorno multipantalla de dimensiones globales que actualmente demanda nuestra sociedad. Es por ello que Alba (2011) advierte la necesidad de que la educación en comunicación audiovisual aproveche la oportunidad de trabajar en el aula una actitud más dialogante y reflexiva del proceso de enseñanza-aprendizaje, con el fin de que permita a los niños y jóvenes valorar de forma consciente su propia actividad como lectores y escritores de discursos mediáticos.

Preparar a los alumnos para que desarrollen estrategias comunicativas y sepan procesar la cantidad de información que se les ofrece hoy es fundamental. Por ello, el sistema educativo debe estar en consonancia con las características de la sociedad para formar a personas en este presente, preparando a las nuevas generaciones en los lenguajes y discursos mediáticos con el fin de, tal y como indican Aguaded y Contín (2002) y Pérez-Tornero (2004), crear ciudadanos reflexivos, críticos, participativos, autónomos y libres. La escuela debe promover esa postura reflexiva, generar espacios para pensar los medios de comunicación y recrear sus productos (Ballesta, 2002), donde los estudiantes participen activamente en el proceso de aprendizaje y colaboren tanto entre ellos como con los profesores trabajando individualmente y en equipo (Freire, 2009). Se trata de formar al ciudadano para que aprenda a utilizar los medios de comunicación de una manera consciente y con capacidad de análisis. Si 
nos detenemos a observar, la sociedad está codificada básicamente con claves audiovisuales o mediáticas, por lo que una educación en medios está totalmente justificada debido a la necesidad de que todos los ciudadanos puedan acceder a esa información, descifrando adecuadamente los códigos y estructuras mediáticas (Aguaded y Guerra, 2012).

La educación para los medios pretende ofertar los instrumentos para expresarse a través de ellos, pues realmente los alumnos pasan más tiempo en contacto con los medios que en la escuela. Los medios de comunicación y las tecnologías digitales de la información conforman un sistema educativo informal que actúa en paralelo a la escuela convencional con sus propios códigos, lenguajes, normas y valores. De ahí que entendamos que tanto la familia como los docentes somos responsables de que los alumnos comprendan el significado de los medios, ya que estos ofrecen formas y contenidos que afectan al sistema de percepción de valores y actitudes que tienen los jóvenes, y les hace asumir como suyos, valores que incluso, en ocasiones, entran en contradicción con los del universo escolar (Aparici, 2005). Observamos así la importancia y necesidad de comprender críticamente el contexto audiovisual y digital en el que nuestros niños y jóvenes viven.

Los medios, al igual que la escuela, se encargan de educar, pero hasta ahora no se ha prestado suficiente atención a cómo lo hacen, por lo que el nuevo tipo de analfabeto es el que tiene dificultades para comprender la realidad construida por los medios. Por tanto, el objetivo de estar alfabetizado es el de comprender y transformar el mundo que le rodea. Los medios contienen conocimiento a través de la actualidad, sus códigos y lenguajes estimulan sensaciones en el usuario (Fontcuberta, 2001), por lo que se aboga, dentro de la alfabetización mediática, por una alfabetización multimedia como la capacidad de descodificar, evaluar y comunicarse en una variedad de medios. Así, mientras hay autores que se decantan por la transdisciplinariedad de esta materia como Fontcuberta (2001), otros como Aparici (2005) establecen las competencias mínimas que debe tener esta asignatura, entre las que destaca: conocer al emisor y la intencionalidad de su mensaje, cómo se logra el fin que persigue, cuáles son los códigos para transmitir el significado, cuáles son los valores del medio, cómo percibe el público la realidad que se les transmite, conocer las técnicas para atraer audiencia, conocer las vinculaciones entre medios e instituciones y sus fuentes de financiación. 


\section{La importancia de la televisión en el entorno escolar}

Los medios de comunicación y en especial la televisión, representan en estos momentos las principales herramientas culturales de nuestra sociedad. Según Sartori (2012), la televisión está transformando al homo sapiens, producto de una cultura basada en la escritura y la lectura, en un homo videns, individuo para el cual la palabra ha quedado destronada por la imagen. Actualmente los niños entran antes en contacto con el medio televisivo, aprendiendo todo lo que de él se deriva, que a leer o a escribir. Se evidencia, de este modo, cómo los medios de comunicación han ido incrementando su valor dentro de la jerarquía social y familiar, consiguiendo penetrar en los hogares, desafiando el control de los padres, y envolviendo al niño en el mundo de los adultos, invitándolo a crecer antes de tiempo. Asimismo, nos imponen hábitos sociales, valores, conductas y nos dan visiones opacas de la realidad que simplemente nos limitamos a aceptar sin someter a ningún tipo de filtro o juicio.

La televisión ha sido y es uno de los medios digitales más discutidos a lo largo de los tiempos. Como indican Muñoz y Pedrero (1996), en su comienzo simplemente era un sistema de trasmisión de información del cuál 'nadie sabía con exactitud cuál sería su destino, ni sus constructores habían estipulado su función, ni la sociedad había fijado un marco de regulación, y ni siquiera los individuos alcanzaban a representar su objeto' (p. 17). Sin embargo, hoy en día, utilizada por un gran número de espectadores, la televisión ha sobrellevado multitud de estudios y ha alcanzado un alto grado de críticas en cuanto a sus repercusiones en la sociedad, especialmente entre los niños y los más jóvenes, siendo tachada, en ocasiones, como la causante de los diferentes fracasos sociales, incluso educativos, que soporta nuestra sociedad y sus miembros.

Conociendo así la importancia del medio televisivo en la vida y desarrollo social y personal de los niños y jóvenes, la escuela, como institución encargada de preparar a nuestros hijos para ser parte integral y activa de la sociedad, tiene la importante función de poner el acento en desarrollar las habilidades críticas y creativas de los niños en relación con este medio (Buckingham, 2008) pues, como Gutiérrez y Tyner (2012, p. 32) exponen, 'la educación, como preparación básica para la vida, no puede pasar por alto ni el entorno donde se desarrolla y que, lógicamente, la condiciona, ni tampoco el modelo cultural y social que desea conseguir'. En este sentido, la escuela no puede volver la vista a un lado 
y seguir instruyendo a sus alumnos para una sociedad que ya no existe, sino que debe capacitarlos para el siglo XXI, para una sociedad movida por las tecnologías y los medios de comunicación, dotándolos de las herramientas e instrumentos necesarios que les permitan una educación integral, así como un desarrollo pleno como ciudadanos activos. Debe otorgarles útiles para la interpretación y uso crítico de los contenidos mediáticos, pues solo así estaremos formando en todas sus dimensiones a los ciudadanos del mañana.

La mayor parte de las ideas e imágenes que los alumnos tienen del mundo les llegan a través de la televisión y de los demás medios, lo que provoca que se acostumbren a aprender de segunda mano sin hacer el esfuerzo de ver y actuar por ellos mismos de una manera autónoma (Alba, 2011). Así, una educación en comunicación mediática es necesaria para alcanzar una correcta alfabetización mediática que, tal como Potter (2014) indica, desarrolle habilidades y estructuras de conocimiento (cognitiva, emocional, moral y estética) relacionadas con el significado de los mensajes que los medios de comunicación transmiten, pues constituye el proceso de enseñar y aprender medios de comunicación, es decir, desarrollar en la persona usuaria de los medios tanto la comprensión crítica como la participativa que la lleve a interpretar y juzgar por sí misma aquello que los medios le ofrecen.

Las experiencias en comunicación audiovisual dentro del aula capacitan a los alumnos para que, como usuarios de los medios y especialmente de aquellos que imponen sus contenidos, como es la televisión, estén en condiciones de interpretar y valorar con criterio sus productos, además de constituir una práctica innovadora y atractiva para el alumnado dentro del aula, que aleje la 'nueva brecha digital' a la que el autor Buckingham (2008) hace referencia cuando se refiere al uso que los niños y jóvenes hacen de la tecnología fuera del aula y al uso que se le da en los centros escolares.

\section{Método}

\section{Diseño}

La investigación presentada se trata de una prospección sobre el uso que se realiza de la televisión en el hogar y en el aula de Educación Primaria. 
Está basada en un diseño no experimental de carácter descriptivo que se limita a observar en su contexto una situación ya existente para analizarla posteriormente.

\section{Participantes}

En esta investigación han participado 320 alumnos de $4^{\circ}$ curso de la etapa de Educación Primaria de edades comprendidas entre los 9 y 10 años, perteneciente a 6 centros educativos con carácter público (52.2\%) y privado/concertado (47.8\%) de la localidad de Molina de Segura (Murcia), ciudad elegida para realizar el trabajo de campo durante el curso académico 2013-14.

Para estimar el tamaño de dicha muestra, teniendo en cuenta el universo total ofrecido por los datos de la Consejería de Educación de la Región de Murcia en el curso académico que se desarrolló la investigación, se aplicó la fórmula para poblaciones finitas de menos de 100.000 elementos de Bugeda, estableciendo un grado de confianza de más del $95 \%$ y un error de estimación menor $\pm 5 \%$ marcado en dicha fórmula (en Jauset, 2000).

\section{Instrumento}

Para la recogida de información se ha utilizado como instrumento un cuestionario anónimo compuesto por 20 preguntas. Los 8 primeros ítems, de respuesta múltiple, están referidos a características personales, académicas, familiares y socioculturales, así como equipamiento y acceso en el hogar. Los 12 siguientes están referidos al uso y consumo de la televisión que el alumno hace en el hogar y al uso del medio en el ámbito escolar. Entre los diferentes ítems encontramos algunas preguntas cerradas de elección única con respuesta dicotómica (si/no) y preguntas categorizadas, algunas de estimación. Algunas de las preguntas son abiertas permitiendo al encuestado expresar sus intereses y opiniones, de manera más detallada, con respuestas de texto libre. Todas ellas centradas en conocer la importancia del uso y consumo de la televisión en el día a día de los alumnos encuestados, así como del uso que se realiza del medio televisivo en las aulas de Educación Primaria y del tiempo dedicado a la formación sobre el medio en la escuela.

La fiabilidad del instrumento analizada a partir del grado de consis- 
tencia interna alfa de Cronbach muestra un valor general de .081, lo que confirma un nivel de consistencia interna adecuado (Cohen, Manion \& Morrison, 2013). Para el tratamiento de los datos obtenidos a partir del cuestionario se utilizó un análisis estadístico descriptivo e inferencial a través del paquete estadístico IBM SSPS Statistics 21.

\section{Procedimiento}

La recogida de datos, bajo la autorización del director de cada centro y de los maestros tutores de aula, fue llevada a cabo durante el horario normal de clase de los centros educativos, utilizando la hora de tutoría en la mayoría de los casos. El muestreo realizado ha sido de tipo aleatorio estratificado en función de centro privado-concertado o público. Una vez definidos los estratos se ha muestreado después cada grupo de manera aleatoria, tomando como elemento muestral las denominadas 'aulas intactas', es decir, los grupos-clase previamente establecidos en cada uno de los centros educativos.

\section{Resultados}

\section{Uso y consumo del medio televisivo}

En relación al tiempo invertido en el acceso a la televisión y su relación con otras preferencias (Figura 1), encontramos que casi la mitad de los alumnos encuestados, un $42.1 \%$, señala 'jugar con los amigos', un $14 \%$ 'estar con la familia' y un 14\% 'ver la televisión'. El resto de alumnos se reparten entre las opciones 'jugar a la videoconsola' (11.5\%), 'leer un libro' (10.8\%) y 'jugar al ordenador' (7.6\%).

Con el fin de conocer si para el alumnado de $4^{\circ}$ de Educación Primaria la televisión era un medio apetecible y valorado se realizó la pregunta ¿te interesa ver la televisión? (Figura 2). Con ella se observa que más de la mitad, 54.6\%, afirma que 'sí' le interesa ver la televisión, seguido de un $24.5 \%$ que señala un interés 'regular' por el medio y un $18.7 \%$ que testifica interesarse 'mucho' por ver la televisión. Asimismo, matizar que también existe un reducido número de alumnos, $2.2 \%$, que no se sienten atraídos por el medio. 


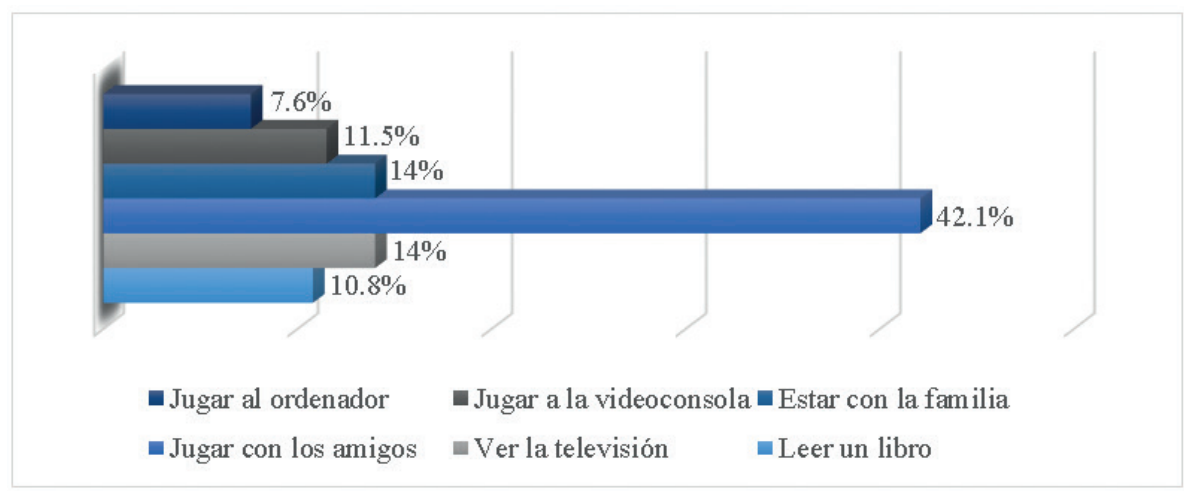

Figura 1. Utilidad del tiempo libre

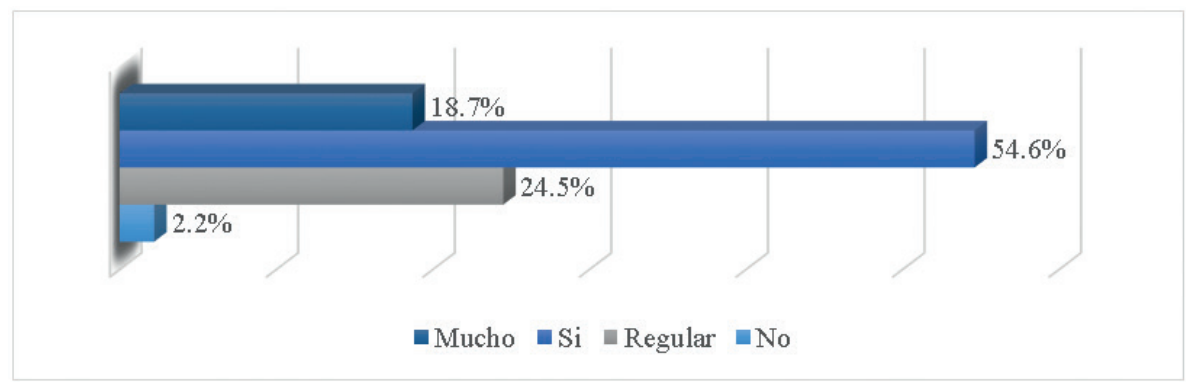

Figura 2. ¿Te interesa ver la televisión?

En relación a la cantidad de horas que el alumnado encuestado pasa frente al medio, a través de la figura 3 se aprecia que cerca del $25 \%$ de los alumnos participantes en la investigación afirma ver la televisión menos de una hora durante los días de semana, reduciéndose esta cifra al $16.5 \%$ durante los días de fin de semana. Asimismo, el $25.9 \%$ de los participantes que han formado parte del estudio explican que ven la televisión una hora al día entre semana y el $25.1 \%$ una hora al día durante los fines de semana. De igual manera, en la figura 3 podemos observar que el $29 \%$ de los niños asegura ver la televisión dos horas al día entre semana, así como el $25.8 \%$ dos horas al día durante los fines de semana. Por último, destacar que el $12 \%$ de los niños encuestados confirma ver más de tres horas la televisión cada día durante la semana y el $22.5 \%$ expresa ver la televisión más de tres horas al día durante los fines de semana. 


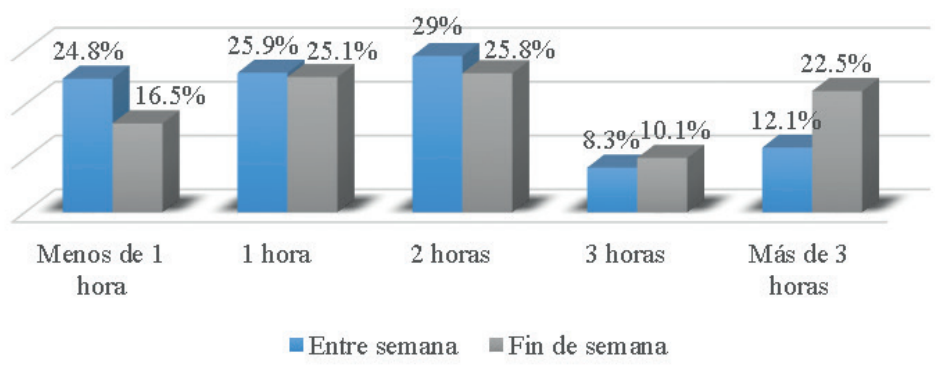

Figura 3. ¿Cuánto tiempo sueles ver la televisión?

\section{Uso del medio televisivo en el aula}

En relación al ámbito escolar, el 30.8\% del alumnado encuestado afirma que sus profesores han utilizado la televisión en clase en algún momento durante el curso escolar, mientras que el $69.2 \%$ niega la utilización del medio por parte del profesor en el aula o en otras actividades del centro escolar.

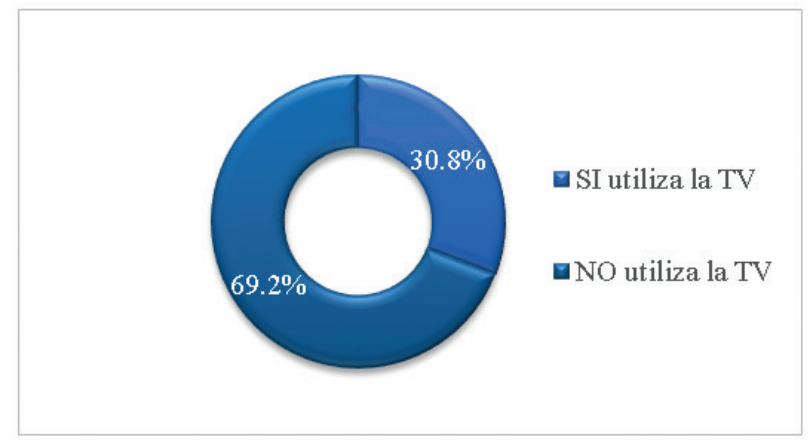

Figura 4. Uso del medio televisivo en el aula de Educación Primaria.

Para establecer una relación entre el uso que el profesor hace del medio televisivo dependiendo del tipo de centro (concertado/privado y público) en que se encuentra escolarizado el alumnado encuestado se ha realizado una prueba Chi-cuadrado de Pearson. Los resultados muestran la no existencia de diferencias estadísticamente significativa $2(1)=.027$ $(p<.05)$ entre los diferentes centros educativos. 


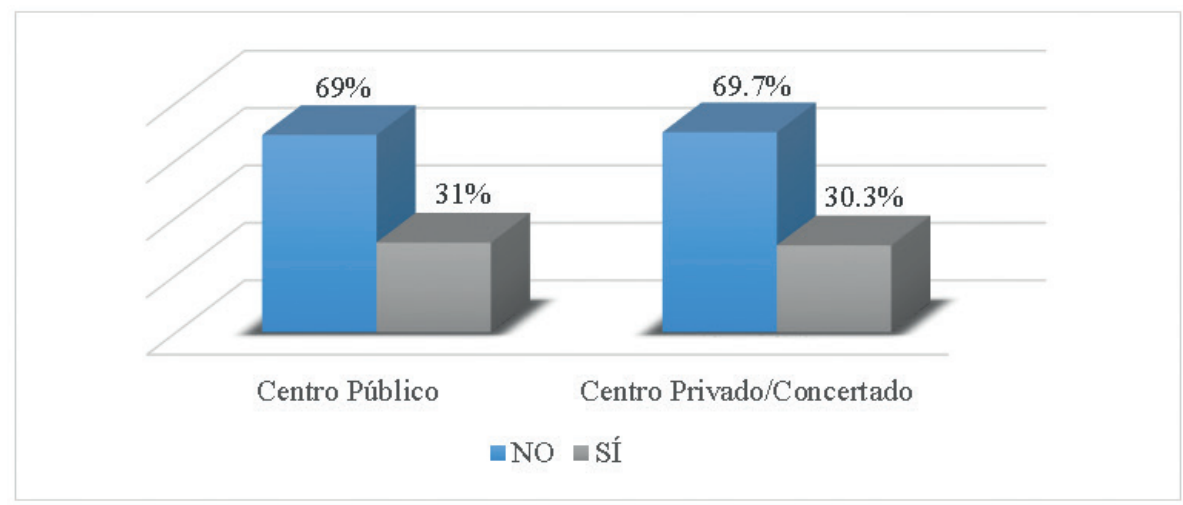

Figura 5. Uso del medio televisivo en el aula de Educación Primaria en función del tipo de centro.

Entre las asignaturas que resultan ser más atrayentes para el profesor a la hora de utilizar el medio televisivo en la exposición y explicación de algunos de sus contenidos destacan, tal como se observa en la Figura 6, las áreas de Ciencias de la Naturaleza (24.25\%), Religión (20.5\%) e Idiomas (15.75\%), seguidas de Ciencias Sociales (6.25\%) y Matemáticas (3.75\%).

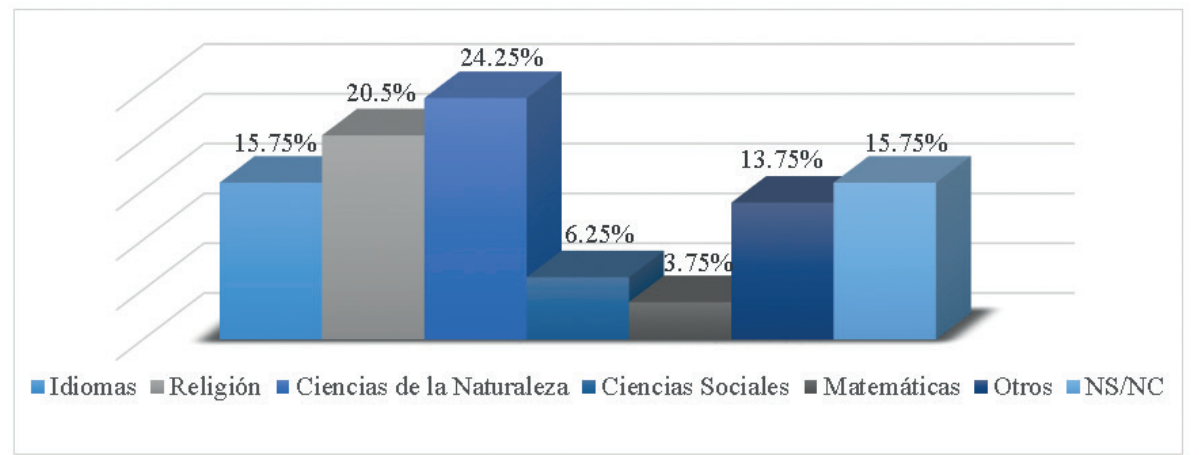

Figura 6. Asignaturas en las que el profesor suele utilizar el medio televisivo en el aula.

En relación a la explotación didáctica que se hace del medio televisivo y tomando como base al alumnado que confirma el uso por parte del profesor en el aula, el $47 \%$ explica que tras el visionado del medio no se ha recibido información alguna de lo visto ni se ha comentado nada al respecto en clase. El 37\%, por el contrario, corrobora sí haber comenta- 
do en clase los contenidos vistos en televisión, así como un 7\% explica que en alguna ocasión han trabajado y comentado los contenidos de la televisión. Un 9\% no sabe y no contesta a la pregunta.

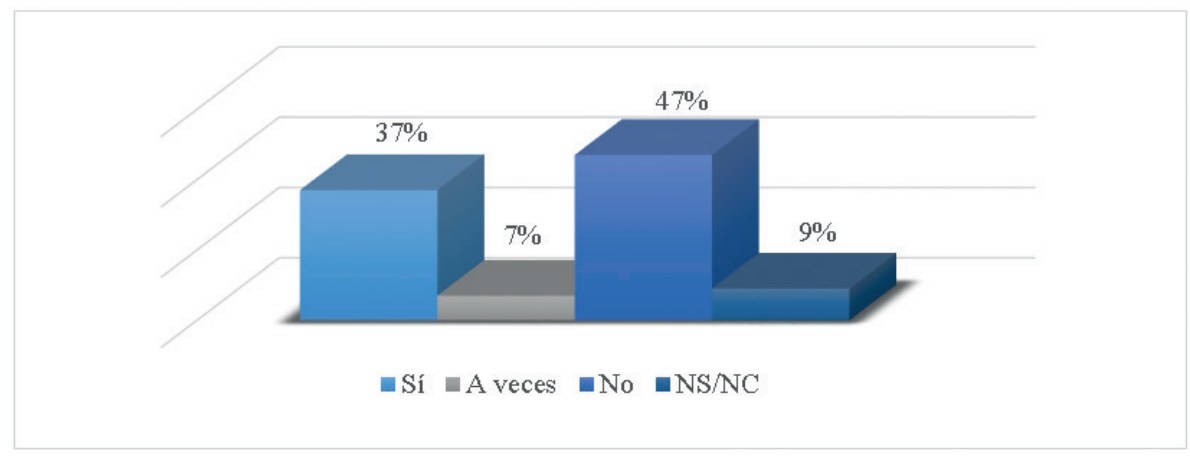

Figura 7. Comentarios y trabajos en el aula sobre los contenidos televisivos.

De igual manera, del porcentaje de alumnos que afirman que sus profesores si utilizan o habían utilizado en clase la televisión, tratamos de relacionar las variables tipo de centro (concertado/privado y público) y el trabajo realizado en clase sobre los programas vistos en televisión durante las sesiones escolares. Para ello llevamos a cabo una prueba t. Los resultados muestran nuevamente la no existencia de diferencias estadísticamente significativa entre los diferentes tipos de centro educativo $(\mathrm{t}(106)=.704, \mathrm{p}<.05)$.

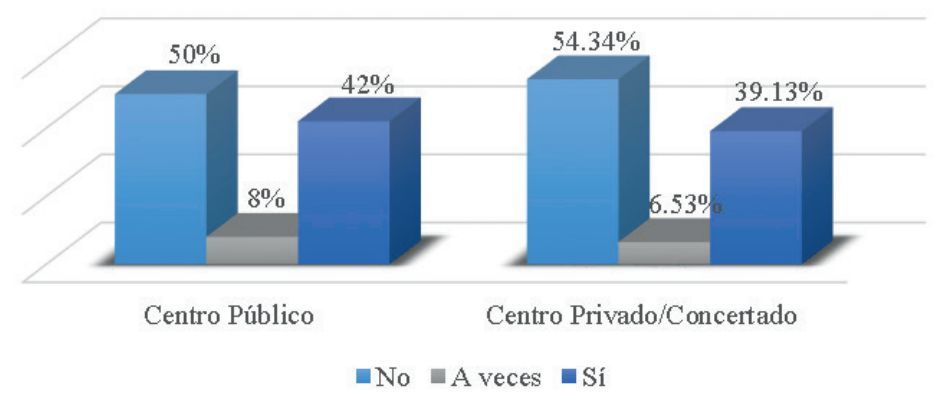

Figura 8. Comentarios y trabajos en el aula sobre los contenidos televisivos en función del tipo de centro. 


\section{Discusión y conclusiones}

Los resultados de la investigación muestran que consumir televisión sigue siendo una de las actividades más desarrollada por los niños cada día. En este sentido, el visionado televisivo durante el tiempo libre es señalado como una de las actividades preferidas por los más jóvenes, llegando a situarse en la segunda opción que ocupa sus horas de ocio. Tras 'jugar con los amigos', el alumnado de entre 9 y 10 años prefiere ver la televisión a leer un libro, jugar con la videoconsola o pasar el rato con el ordenador, aspecto interesante si destacamos que estos últimos se corresponden con medios y tecnologías más modernas que el televisor. Según nuestro estudio, cerca de un 30\% del alumnado confirma ver la televisión aproximadamente 2 horas al día, así como casi un 21\% lo hace tres o más de tres horas durante los días de semana, consumo que se incrementa durante el fin de semana, donde aproximadamente un $33 \%$ de los niños confirma su visionado durante 3 o más horas al día. Estos hechos igualmente se observan en investigaciones previas (Del Moral y Villalustre, 2010; Del Moral, Villalustre y Neira, 2010; Perlado y Sevillano, 2003) que ponen de manifiesto que la dedicación de los escolares a la televisión y videojuegos ocupa más del 38\% de su tiempo libre.

El estudio realizado nos revela igualmente la existencia de una tendencia generalizada, por parte del profesorado de la etapa de Educación Primaria, tanto los centros de carácter público, privado y concertado, de escaso uso de la televisión en el aula para el desarrollo de contenidos curriculares, destacando su limitado visionado en asignaturas como Religión, Idiomas o Ciencias de la Naturaleza. Asimismo, destaca la escasa explotación didáctica sobre los contenidos televisivos visionados entre aquellos docentes que sí la utilizan en el aula.

En conclusión, si tenemos en cuenta que la televisión es uno de los medios de comunicación más utilizados por los niños en el día a día, este escaso uso que se realiza de la misma desde el aula escolar permite evidenciar la gran separación entre escuela y sociedad (nueva brecha digital) y, por tanto, el absurdo que los niños viven en relación a los modelos educativos y procesos de enseñanza-aprendizaje desarrollados en la escuela y sus actividades fuera de la misma (Buckingham, 2011). Será, en consecuencia, necesario conectar esos procesos de enseñanzaaprendizaje que tienen lugar dentro de la institución escolar con las necesidades de los estudiantes de hoy día, ayudándoles a adquirir las 
herramientas necesarias para enfrentarse a los grandes retos y problemas que la sociedad actual les presenta (De Pablos, Colás y Villaciervos, 2010). Debemos, por tanto, concienciar a los maestros de la importancia de formar a los niños en y para la sociedad que les espera, de la que son y serán parte integrante y fundamental, y para la que queremos sean ciudadanos activos, reflexivos y críticos, pues, ¿no es esta la verdadera finalidad de la escuela? Igualmente debemos sensibilizar a los maestros de que todos los medios de comunicación, en este caso la televisión, pueden ser convertidos en herramientas de servicio educativo cuando la educación los utiliza de manera adecuada con fines formativos e instructivos. Asimismo, debemos dar a conocer que todo tipo de programas televisivos pueden adquirir virtud educadora si el propio maestro sabe trabajarlos en el aula, ayudando al alumno a descodificar los mensajes, siempre desde un planteamiento pedagógico, incitando a la reflexión de los contenidos, así como de las acciones y actitudes observadas en el medio. Para ello, como comentamos, será necesario que también los maestros, como usuarios y consumidores de los medios en general y más concretamente de la televisión, desarrollen un conocimiento previo y una serie de competencias que permita revalorizar 'el poder de los medios como elementos para el desarrollo de la cultura popular y el fortalecimiento de la ciudadanía', esto es 'recuperar la función social de la cultura y los medios de producción a través de la práctica pedagógica cotidiana' (Cuervo y Medrano, 2013, p. 123).

Los alumnos y maestros tienen en sus manos un instrumento para empezar a desafiar la gran desigualdad de conocimiento y poder que existe entre los que fabrican la información y quienes simplemente la consumen. Por tanto, ante el consumo televisivo, la educación tiene que desarrollar propuestas relacionadas con una formación en materia de comunicación que suponga integrar el contenido de sus programas, el análisis de sus propuestas y comprender así qué está pasando en la actualidad. Consideramos además que estudiar los medios de comunicación, en este caso la televisión, creando en el aula espacios para su reflexión y disfrute, favorece tanto al alumno como al maestro, ya que el alumno se interesa porque le hablas de su mundo y se siente más motivado, y el maestro porque sabe que estudiarlos conlleva numerosos beneficios educativos y éticos de gran importancia para sus alumnos (Alba, 2011). Así, no debemos implantar las tecnologías y los medios en la escuela de una manera simplemente instrumental, no debemos ense- 
ñar a usarlos sin más, sino que debemos ir en dirección a una verdadera 'alfabetización mediática' que permita a los niños y jóvenes conocer y comprender adecuadamente todos los procesos comunicativos en los que la sociedad de hoy día los envuelve, haciéndolos capaces de apropiarse de ellos y emplearlos de manera creativa y activa como canales de comunicación propios. De esta manera, aprenderán, además de a usarlos de manera crítica, a crear a partir de ellos.

\section{Referencias}

Aguaded, J. I. (2010). La educación para la comunicación. Nueva alfabetización para un mundo global. En M. E. Del Moral. (Coord.), Televisión: desarrollo de la creatividad e infancia (pp. 55-70). Barcelona, España: Octaedro.

Aguaded, J. I. y contín, S. (2002). Jóvenes, aulas y medios de comunicación. Propuestas y prácticas mediáticas para el aula. Buenos Aires, Argentina: Ediciones Ciccus.

Aguaded, J. I., y Guerra, S. (2012). Razones para una educación mediática en la sociedad multipantallas. Sphera Pública, 12, 21-39.

Alba, R. B. (2011). 10 ideas clave. Educar en medios de comunicación. La educación mediática. Barcelona, España: Grao

Álvarez, G. (2012). Las nuevas tecnologías en el contexto universitario: sobre el uso de blogs para desarrollar las habilidades de lectoescritura de los estudiantes [New Technologies in the University Context: The Use of Blogs for Developing Students' Reading and Writing Skills]. RUSC. Universities and Knowledge Society Journal, 9(2), 3-17.

Aparici, R. (2005). Medios de comunicación y educación. Revista de educación, 338, 85-99.

Ballesta, J. (Coord.) (2002). Medios de comunicación para una sociedad global. Murcia, España: Servicio de publicaciones de la Universidad de Murcia.

Ballesta, J. (2009). Formar hoy con los medios de comunicación. En J. De Pablos (Coord.), Tecnología Educativa. La formación del profesorado en la era de Internet (pp. 425447). Málaga, España: Aljibe.

Ballesta, J. (2010). Para comprender los contenidos mediáticos. En J. Peirats y Á. San Martín (Coord.), Tecnologías educativas 2.0. Didáctica de los contenidos digitales (pp. 47-64). Madrid, España: Pearson.

Ballesta, J., Lozano, J y Cerezo, M. (2014). El uso y consumo de TIC en el alumnado autóctono y extranjero de Educación Secundaria Obligatoria de la Región de Murcia. RED, Revista de Educación a Distancia, 41, 1-32.

Buckingham, D. (2008). Repensar el aprendizaje en la era de la cultura digital. El monitor, 18, 27-30.

Buckingham, D. (2011). Media Education: Literacy, Learning and Contemporary Culture. Cambridge, England: Polity Press.

Burbules, N. (2007). Riesgos y promesas de las TIC en la educación: ¿Qué hemos apren- 
El uso de la televisión en el hogar y en el aula de Educación Primaria

dido en estos diez últimos años? En C. Magadán y V. Kelly (Eds.), Las TIC. Del aula a la agenda pública (pp. 31-40). Buenos Aires, Argentina: Fondo de las Naciones Unidas para la Infancia / UNICEF.

Cohen, L., Manion, L. y Morrison, K. (2013). Research methods in education. London \& New York: Routledge.

Cuervo, S. L., y Medrano, C. (2013). Alfabetizar en los medios de comunicación: más allá del desarrollo de competencias. Teoría de la Educación. Revista Interuniversitaria, 25(2), 111-131.

De la Torre, A. (2009). Nuevos perfiles en el alumnado: la creatividad en nativos digitales competentes y expertos rutinarios. Revista de Universidad y Sociedad del Conocimiento, 6(1), 7-14.

De Pablos, J., Colás, P. y Villaciervo, P. (2010). Políticas educativas, buenas prácticas y TIC. Education in the Knowledge Society (EKS), 11(1), 180-202. Recuperado de https://goo.gl/rT6jw9

Del Moral, M. E., y Villalustre, L. (2010). Consumo televisivo y de videojuegos de los escolares asturianos versus desarrollo de competencias audiovisuales y digitales. En E. del Moral. (Coor.), Televisión: desarrollo de la creatividad e infancia (pp. 31-46) Barcelona, España: Octaedro.

Del Moral, M. E., Villalustre. L., y Neira, M. R. (2010). La asimilación cognitiva infantil de los estereotipos presentados a través de los dibujos animados. Observatorio (OBS), 4(3).

Fontcuberta, M. (2001). Comunicación y educación, una relación necesaria. Cuadernos de Información, 14, 141-147.

Freire, J. (2009). Cultura digital y prácticas creativas en educación. Revista de Universidad y Sociedad del Conocimiento, 1, 2-6.

Gutiérrez, A., y Tyner, K. (2012). Educación para los medios, alfabetización mediática y competencia digital [Media Education, Media Literacy and Digital Competence] Comunicar: Revista científica iberoamericana de comunicación y educación, 38(19), 31-39.

Gómez, Á. H., y Aguaded, J. I. (2011). Recomendaciones para el desarrollo de la alfabetización mediática en Brasil: propuestas desde la experiencia europea. Resgate: Revista Interdisciplinar de Cultura, 19(2), 03-15.

Jauset, J. A. (2000). La investigación de audiencias en televisión: fundamentos estadísticos. Barcelona, España: Paidós.

Koltay, T. (2011). The Media and the Literacies: Media Literacy, Information Literacy, Digital Literacy. Media, Culture \& Society, 33(2), 211-221.

Masterman, L. (1993). La enseñanza de los medios de comunicación. Madrid, España: De la Torre.

Middaugh, E., y Kahne, J. (2013). Nuevos medios como herramienta para el aprendizaje cívico [New Media as a Tool for Civic Learning]. Comunicar: Revista científica iberoamericana de comunicación y educación, 40(10) 99-108.

Muñoz, J. J. y Pedrero, L. M. (1996) La televisión y los niños. Salamanca, España: Cervantes.

Núñez, T. (2009). Las familias y los medios de comunicación. En T. Loscertales y T. 
Núñez. (coords.), Familias y medios de comunicación. Propuestas para un consumo responsable y evitar la adicción (pp. 8-36). Sevilla, España: Eduforma.

Pérez-Tornero, J.M. (2004). Promoting Digital Literacy. Understanding Digital Literacy. Barcelona, España: Universidad Autónoma de Barcelona.

Perlado, L. y Sevillano, M. L. (2003). La influencia de la televisión en los niños. Enseñanza, 21, 163-178.

Potter, W. J. (2014). Media literacy. California, EEUU: Sage Publications.

Sartori, G. (2012). Homo videns. Alicante, España: Santillana Ediciones.

Sevillano, M. L. (2012). Estilos de enseñar y aprender con investigación y uso de medios de comunicación. En R. Sánchez, P. Isla y F. J. Sánchez. (Coords.), Medios de comunicación en el aula. Enfoques y perspectivas de trabajo (pp. 11-26). Barcelona, España: Octaedro. 
